\title{
Method for Evaluating the Statistical Relationship between Sound Pressure Level and Noise Annoy- ance Based on a Nonlinear Time Series Regression Model and an Experiment
}

\author{
Hisako Orimoto, Akira Ikuta and Yegui Xiao \\ Department of Management and Information Systems, Prefectural University of Hiroshima, 1-1-71 Ujina-Higashi, \\ Minamiku, Hiroshima 734-8558, Japan
}

(Received 29 October 2013; accepted 25 August 2015)

\begin{abstract}
Identifying the quantitative relationship between the sound pressure level and noise annoyance for environmental noises is important from the viewpoint of noise assessment. In this study, a method for predicting the probabilistic evaluation quantities like $L_{x}\left((100-x)\right.$ percentile level) and $L_{A e q}$ (equivalent A-weighted sound pressure level) of the noise environment is proposed by introducing a nonlinear time series regression model between the sound pressure level and noise annoyance. More specifically, the joint probability distribution is expanded in an orthonormal expansion series in which linear and nonlinear correlation information is reflected hierarchically in each expansion coefficient. Next, statistical methods for predicting the sound pressure level and the noise annoyance are proposed by introducing a nonlinear time series regression model based on the above probability distribution. The validity of the proposed method is confirmed by applying it to a set of instantaneous data on sound pressure level and noise annoyance observed in a real sound environment.
\end{abstract}

\section{INTRODUCTION}

Identifying the quantitative relationship between the sound pressure level and noise annoyance for environmental noise is important from the viewpoint of noise assessment. ${ }^{1-3}$ Usually, an investigation based on the questionnaires is performed, as the experimental measurement at all points in the entire area of the regional sound environment is difficult. Furthermore, statistical sound evaluation quantities, such as $L_{x}$ based on the probability distribution of sound pressure level and $L_{A e q}$ based on averaged energy of sound pressure level, are widely used in the evaluation of the sound environment. Therefore, it is very important to determine the relationship between the noise annoyance and the sound pressure level from a statistical point of view.

In a previous study, a state estimation method was proposed for the fluctuation waveform of the sound pressure level by time-dependent sound pressure level based on the observation data of noise annoyance from the viewpoint of systems theory. ${ }^{4}$ The relationship of the sound pressure level and noise annoyance was regarded as the input and output of a fuzzy probability system with uncertainty and vagueness. A method was theoretically derived for estimating the fluctuation waveform of the sound pressure level or the system input by use of the observation data of the noise annoyance or the system output. In analyses of environmental noise, two approaches can be considered. One is analysis from the bottom-up viewpoint, structurally based on the fundamental mechanism on the relation- ship between noise annoyance and sound pressure level. The other is the top-down method, which is connected with evaluation of environmental noise in the case of unknown structural mechanism. Since the analysis method considering the physical mechanism from the bottom-up viewpoint was adopted in the previous study, the derivation process of the estimation algorithm was rather complicated.

On the other hand, a method based on the top-down viewpoint can be proposed by regarding time-dependent sound pressure level and noise annoyance as the resultant time series data and by considering their mutual correlation information. In this paper, a practical evaluation method is proposed, which is simple in form as compared with the previous study. The joint probability distribution for the sound pressure level and noise annoyance is first considered for the purpose of using the usual liner correlation as well as the higher order nonlinear correlation information between both variables. Next, two probabilistic methods are proposed based on the joint probability distribution in an orthonormal expansion series ${ }^{5}$, where linear and nonlinear correlation information is reflected hierarchically in each expansion coefficient. One method predicts the noise annoyance based on the observation of sound pressure level, and the other is a prediction for the sound pressure level from the noise annoyance. Finally, the effectiveness of the proposed methods are confirmed by applying them to a psychological experiment with the road traffic noise, where the linear regression model and neural network are compared. 


\section{THEORETICAL CONSIDERATION}

\subsection{Prediction of Noise Annoyance by Observing Sound Pressure Level}

\subsubsection{Prediction of noise annoyance based on con- ditional probability distribution}

The sound pressure level in the actual sound environment exhibits complex fluctuation pat-tern. For example, there are various linear and nonlinear correlations among several instantaneous values. It has been reported in psychological acoustics that the human psychological evaluation for noise annoyance can be distinguished up to 7 scores: 1-Very calm, 2-Calm, 3Mostly calm, 4-Little noisy, 5-Noisy, 6-Fairly noisy, 7-Very noisy. ${ }^{6}$

Let $x$ be the sound pressure level and $y$ be the noise annoyance score in human evaluation (i.e. $y=1,2, \cdots, 7$ ). Furthermore, the sound pressure levels at $p$ past discrete time for $y$ at an arbitrary discrete time are expressed as $x_{p+1}(p=$ $0,1,2, \cdots)$. The noise annoyance can be predicted recursively on the basis of the sound pressure levels $x_{1}, x_{2}, \cdots, x_{p}$ by adopting $y$ so as to maximize the conditional probability $P\left(y \mid x_{1}, x_{2}, \cdots, x_{p}\right)$ as the prediction of $y$. First, the joint probability distribution $P(y, \mathbf{x})$ of $y$ and $\mathbf{x}\left(=\left(x_{1}, x_{2}, \cdots x_{p}\right)\right)$ is expanded into an orthonormal polynomial series based on the product of the standard probability distribution for each variable. $^{7}$

$$
\begin{aligned}
& P(y, \mathbf{x})=P_{0}(y) P_{0}\left(x_{1}\right) P_{0}\left(x_{2}\right) \cdots \\
& \cdots P_{0}\left(x_{p}\right) \sum_{m=0}^{\infty} \sum_{n_{1}=0}^{\infty} \cdots \sum_{n_{p}=0}^{\infty} A_{m n_{1} \cdots n_{p}} \varphi_{m}(y) \phi_{n_{1}}\left(x_{1}\right) \cdots \\
& \cdots \phi_{n_{p}}\left(x_{p}\right) . \\
& A_{m n_{1} \cdots n_{p}}=\left\langle\varphi_{m}(y) \phi_{n_{1}}\left(x_{1}\right) \phi_{n_{2}}\left(x_{2}\right) \cdots \phi_{n_{p}}\left(x_{p}\right)\right\rangle ; \\
& \phi_{n_{i}}\left(x_{i}\right)=\frac{1}{\sqrt{n !}} H_{n}\left(\frac{x_{i}-\mu_{x_{i}}}{\sigma_{x_{i}}}\right) ; \\
& \phi_{m}(y)=\frac{1}{\sqrt{\left(\frac{N-M}{h}\right)^{(m)} m !}}\left(\frac{1-p}{p}\right)^{\frac{m}{2}} \frac{1}{h^{m}} \sum_{j=0}^{m}\left(\begin{array}{c}
m \\
j
\end{array}\right) \\
& (-1)^{m-j}\left(\frac{p}{1-p}\right)^{m-j}(N-y)^{(m-j)}(y-M)^{(j)} ;
\end{aligned}
$$

$$
y^{(j)}=y(y-h)(y-2 h) \cdots(y-(n-1) h), \quad y^{(0)}=1,
$$

where $<>$ denotes the averaging operation with respect to the variables. Two functions $\phi_{n_{i}}\left(x_{i}\right), \varphi_{m}(y)$ are orthonormal polynomials with the weighting functions $P_{0}\left(x_{i}\right)$ and $P_{0}(y)$ are obtained. ${ }^{5} H_{n}(\bullet)$ denotes the Hermite polynomial with $n$th order, and $y^{(j)}$ is the $j$ th order factorial function. ${ }^{5}$ The Gaussian distribution is adopted as the standard probability distributions of $P_{0}\left(x_{i}\right)$ for the continuous variable $x_{i}$, and the generalized binomial distribution is adopted as $P_{0}(y)$ for the quantized value $y$.

$$
\begin{gathered}
P_{0}\left(x_{i}\right)=\frac{1}{\sqrt{2 \pi \sigma_{x_{i}}^{2}}} e^{-\frac{\left(x_{i}-\mu_{x_{i}}\right)^{2}}{2 \sigma_{x_{i}}^{2}}} ; \\
\mu_{x_{i}}=<x_{i}>, \quad \sigma_{x_{i}}^{2}=<\left(x_{i}-\mu_{x_{i}}\right)^{2}>; \\
P_{0}(y)=\frac{\left(\frac{N-M}{h}\right) !}{\left(\frac{y-M}{h}\right) !\left(\frac{N-y}{h}\right) !} p^{\frac{y-M}{h}}(1-p)^{\frac{N-y}{h}} ; \\
p=\left(\mu_{y}-M\right) /(N-M), \quad \mu_{y}=<y>;
\end{gathered}
$$

where $N, M$ and $h$ are defined as follows:

$$
\begin{aligned}
N=7 ; & \text { the maximum value of } y, \\
M=1 ; & \text { the minimum value of } y, \\
h=1 ; & \text { the level difference interval of } y .
\end{aligned}
$$

The linear and nonlinear correlation information between $y$ and $\mathrm{x}$ is reflected hierarchically in each expansion coefficient $A_{m n_{1} \cdots n_{p}}$. Next, an expansion series expression on the conditional proba-bility distribution can be obtained from Eq. 1, as follows:

$$
\begin{aligned}
& P(y \mid \mathbf{x})=\frac{P(y, \mathbf{x})}{P(\mathbf{x})}= \\
& \frac{P_{0}(y) \sum_{m=0}^{\infty} \sum_{n_{1}=0}^{\infty} \cdots \sum_{n_{p}=0}^{\infty} A_{m n_{1} \cdots n_{p}} \phi_{m}(y) \varphi_{n_{1}}\left(x_{1}\right) \cdots \varphi_{n_{p}}\left(x_{p}\right)}{\sum_{n_{1}=0}^{\infty} \cdots \sum_{n_{p}=0}^{\infty} A_{0 n_{1} \cdots n_{p}} \varphi_{n_{1}}\left(x_{1}\right) \cdots \varphi_{n_{p}}\left(x_{p}\right)} .
\end{aligned}
$$

By substituting Eqs. (3)-(9) into Eq. (10), the conditional probability distribution can be obtained. Moreover, the probability distribution of noise annoyance score $y$ can be evaluated by averaging the conditional probability distribution $P(y \mid \mathbf{x})$ in Eq. (10) by using time series data of sound pressure level as follows:

$$
\begin{aligned}
& P(y)=P_{0}(y) \sum_{m=0}^{\infty} \\
& \left\langle\frac{\sum_{n_{1}=0}^{\infty} \cdots \sum_{n_{p}=0}^{\infty} A_{m n_{1} \cdots n_{p}} \varphi_{n_{1}}\left(x_{1}\right) \cdots \varphi_{n_{p}}\left(x_{p}\right)}{\sum_{n_{1}=0}^{\infty} \cdots \sum_{n_{p}=0}^{\infty} A_{0 n_{1} \cdots n_{p}} \varphi_{n_{1}}\left(x_{1}\right) \cdots \varphi_{n_{p}}\left(x_{p}\right)}\right\rangle \varphi_{m}(y) .
\end{aligned}
$$

\subsubsection{Prediction of noise annoyance based on multi- dimensional nonlinear regression model}

The linear and nonlinear correlation information between $y$ and $\mathbf{x}$ is all included in condi-tional probability distribution $P(y \mid \mathbf{x})$. Especially, when predicting $y$ based on $\mathbf{x}$, the regression function defined as the expectation of $y$ conditioned by $\mathbf{x}$ can be adopted as the prediction of $y$.

$$
\hat{y}=\langle y \mid \mathbf{x}\rangle=\sum_{y=1}^{7} y \cdot P(y \mid \mathbf{x}) .
$$


After substituting Eq. (10) into Eq. (12), by taking into consideration the orthonormal condition of orthonormal polynomial $\varphi_{m}(y)$ :

$$
\sum_{y=1}^{7} P_{0}(y) \varphi_{n}(y) \varphi_{m}(y)=\delta_{n m}
$$

the regression function $\hat{y}$ is given by Eq. (14) (top of the next page), where $d_{1 m}$ are coefficients in the orthogonal expansion of $y$ :

$$
y=\sum_{m=0}^{1} d_{1 m} \varphi_{m}(y)
$$

and can be derived as follows:

$$
\begin{aligned}
& d_{11}=\sqrt{(N-M) h p(1-p)}, \\
& d_{10}=(N-M) p+M .
\end{aligned}
$$

\subsection{Prediction of Sound Pressure Level by Observing Noise Annoyance}

The sound pressure level at an arbitrary discrete time is predicted by observing the noise annoyance scores $\mathbf{y}$ (= $\left.y_{1}, y_{2}, \cdots, y_{q}\right)$ up to $(q-1)$ past discrete time based on the multi-dimension nonlinear regression model. Considering the orthonormal condition of the polynomial function

$$
\int_{-\infty}^{\infty} P_{0}(x) \phi_{m}(x) \phi_{n}(x) d x=\delta_{m n} ;
$$

and using the same calculation process as given in Section 2.1.2, the prediction $\hat{x}$ of sound pres-sure level $x$ is given by Eq. (17) (top of the next page), where the coefficients $c_{1 m}$ are specifically given in the orthogonal expansion $x=\sum_{m=0}^{1} c_{1 m} \phi_{m}(x)$, as $c_{11}=\sigma_{x_{i}}, c_{10}=\mu_{x_{i}}$. Furthermore, the expansion coefficients $B_{m n_{1} n_{2} \cdots n_{q}}$ are defined as follows:

$$
B_{m n_{1} n_{2} \cdots n_{q}}=\left\langle\phi_{m}(x) \varphi_{n_{1}}\left(y_{1}\right) \varphi_{n_{2}}\left(y_{2}\right) \cdots \phi_{n_{q}}\left(y_{q}\right)\right\rangle .
$$

\section{EXPERIMENTAL CONSIDERATION}

The road traffic noise with frequency characteristic of broadband was recorded at a position being $1 \mathrm{~m}$ apart from one side of the national road by use of a sound pressure level meter and a data recorder. Two kinds of data (i.e., data 1 and data 2) for the sound pressure level of road traffic noise in two typical cases of light and heavy traffic flow with mean values of $71.4[\mathrm{~dB}]$ and $80.2[\mathrm{~dB}]$ were measured by using sound pressure level meter (model NL-06 integral standard type, Rion Co.) under an A-characteristic and FAST response with a time constant of 0.125 seconds in an RMS circuit. By replaying the recorded data through amplifier and loudspeaker in a laboratory room, 6 subjects between the ages of 22-24 with normal hearing ability judged one score among 7 noise annoyance scores $^{6}$ at every 5 seconds. The experiment was conducted in a laboratory room, and the effect of the background noise could be ignored.

The proposed study is focused on the derivation of a new method to predict the sound pres-sure level and noise annoyance from the theoretical viewpoint. Since the purpose of the study is to suggest a theoretical method for estimation, the usefulness of the proposed method was confirmed by applying it to 6 subjects.

\subsection{Experimental Consideration for Prediction of Noise Annoyance by Observing Sound Pressure Level}

\subsubsection{Comparison of the proposed method based on conditional probability distribution and neural network}

Two cases with and in Eq. (10) were considered:

$$
\begin{gathered}
P\left(y \mid x_{1}\right)= \\
\frac{P_{0}(y) \sum_{m=0}^{i} \sum_{n=0}^{i} A_{m n} \varphi_{m}(y) \phi_{n}\left(x_{1}\right)}{\sum_{n=0}^{i} A_{0 n} \phi_{n}\left(x_{1}\right)}, \quad(p=1) \\
\frac{P\left(y \mid x_{1}, x_{2}\right)=}{P_{0}(y) \sum_{m=0}^{i} \sum_{n_{1}=0}^{i} \sum_{n_{2}=0}^{i} A_{m n_{1} n_{2}} \varphi_{m}(y) \varphi_{n_{1}}\left(x_{1}\right) \varphi_{n_{2}}\left(x_{2}\right)} \\
\sum_{n_{1}=0}^{i} \sum_{n_{2}=0}^{i} A_{0 n_{1} n_{2}} \phi_{n_{1}}\left(x_{1}\right) \phi_{n_{2}}\left(x_{2}\right)
\end{gathered}
$$

Though the conditional probability distribution in Eq. (19) is expressed in an infinite expansion series, only finite expansion coefficients can be used in the application to real noise environment. The expansion coefficients $A_{m n_{1} n_{2}}$ and $A_{0 n_{1} n_{2}}$ in Eq. (19) with $m \leq i, n_{1} \leq i$ and $n_{2} \leq i(i=1,2, \cdots, 6)$ were considered in the experiment. First, these expansion coefficients were calculated by use of data 1 as the learning data. Next, the noise annoyance score was predicted by observing the sound pressure level of data 2 as the prediction data. The prediction results are shown in Table 1 as the recognition rate. In an ideal case with infinite numbers of data, the recognition rate gets better with increasing order. However, a recognition rate sometimes decreases with increasing order because excess order includes unnecessary information in real cases with finite numbers of available data. Therefore, the optimal order exists, and finding the optimal order becomes an important issue for future development.

In this study, the best recognition rates were obtained in the case that $p=1, i=4$ and $p=2, i=2$. One of the prediction results is shown in Fig. 1 in the case that $p=1$ and $i=4$. In this experiment, the subjects judged annoyance scores from 3 to 7 among 7 scores by hearing the replayed road traffic noise 


$$
\begin{gathered}
\hat{y}=\frac{\sum_{m=0}^{1} \sum_{n_{1}=0}^{\infty} \sum_{n_{2}=0}^{\infty} \cdots \sum_{n_{p}=0}^{\infty} A_{m n_{1} n_{2} \cdots n_{p}} d_{1 m} \phi_{n_{1}}\left(x_{1}\right) \phi_{n_{2}}\left(x_{2}\right) \cdots \phi_{n_{p}}\left(x_{p}\right)}{\sum_{n_{1}=0}^{\infty} \sum_{n_{2}=0}^{\infty} \cdots \sum_{n_{p}=0}^{\infty} A_{0 n_{1} n_{2} \cdots n_{p}} \phi_{n_{1}}\left(x_{1}\right) \phi_{n_{2}}\left(x_{2}\right) \cdots \phi_{n_{p}}\left(x_{p}\right)} \\
\hat{x}=\langle x \mid \mathbf{y}\rangle=\frac{\sum_{m=0}^{1} \sum_{n_{1}=0}^{\infty} \sum_{n_{2}=0}^{\infty} \cdots \sum_{n_{q}=0}^{\infty} B_{m n_{1} n_{2} \cdots n_{q}} c_{1 m} \varphi_{n_{1}}\left(y_{1}\right) \varphi_{n_{2}}\left(y_{2}\right) \cdots \varphi_{n_{q}}\left(y_{q}\right)}{\sum_{n_{1}=0}^{\infty} \sum_{n_{2}=0}^{\infty} \cdots \sum_{n_{q}=0}^{\infty} B_{0 n_{1} n_{2} \cdots n_{q}} \varphi_{n_{1}}\left(y_{1}\right) \varphi_{n_{2}}\left(y_{2}\right) \cdots \varphi_{n_{q}}\left(y_{q}\right)} .
\end{gathered}
$$

Table 1. Comparison of the recognition rate between the proposed method by conditional probability distribution and neural network. The cells with highest recognition rate are highlighted.

\begin{tabular}{||c|c|c|}
\hline \multirow{2}{*}{$\begin{array}{c}\text { Order } \\
(i)\end{array}$} & \multicolumn{2}{|c|}{ Proposed Method } \\
\cline { 2 - 3 } & $p=1$ & $p=2$ \\
\hline 1 & $71.7 \%$ & $68.9 \%$ \\
\hline 2 & $68.3 \%$ & $71.4 \%$ \\
\hline 3 & $73.3 \%$ & $68.1 \%$ \\
\hline 4 & $76.7 \%$ & $70.6 \%$ \\
\hline 5 & $71.7 \%$ & $68.1 \%$ \\
\hline 6 & $69.2 \%$ & $63.9 \%$ \\
\hline \multicolumn{3}{|c|}{ Neural Network } \\
\hline- & $p=1$ & $p=2$ \\
\hline- & $42.5 \%$ & $42.0 \%$ \\
\hline
\end{tabular}

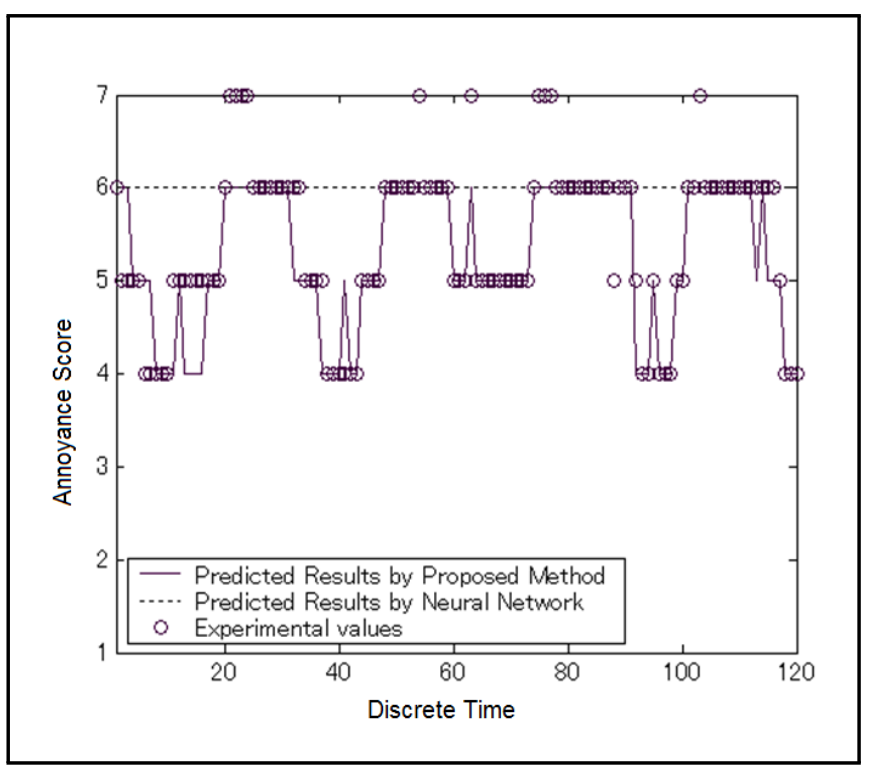

Figure 1. A comparison between the predicted results by the conditional probability distribution and the neural network.

of data 1 . Similarly, the result from 4 to 7 among 7 scores by hearing of data 2 was obtained. For comparison, the prediction results by the neural network, which is frequently used in the field of pattern recognition, were compared with the proposed method. The neural network of three-layer structure with the sigmoid function in the middle layer was adopted and back propagation was used as the learning algorithm. In the learning process of the neural network, 40 different initial values were given to decide the synaptic weight. The number of units in a middle layer varied from 2 to 20 , and the same procedures were repeated. After the repeated estimation processes, the weight with best recognition rate was adopted. The proposed

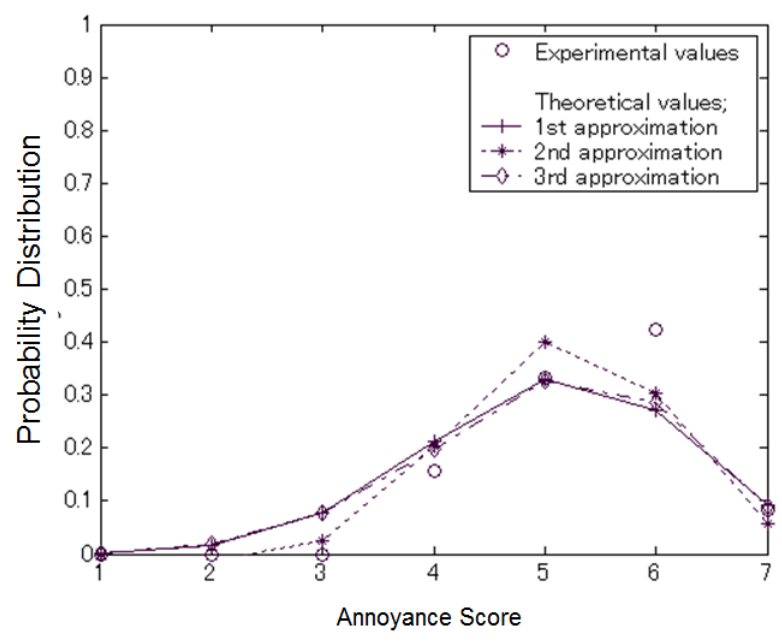

Figure 2. Predicted result for the probability distribution of noise annoyance.

method shows more accurate prediction for the noise annoyance than the neural network. Furthermore, the predicted result for the probability distribution of noise annoyance score based on the observed data of sound level is shown in Fig. 2 in the case that $p=1$ and $i=4$. In this figure, the experimental values were obtained directly by calculating the frequency distribution based on the evaluation data of noise annoyance scores by 6 subjects. On the other hand, the theoretical values were predicted by using Eq. (11) based on the measured data of sound pressure level. The theoretically-predicted probability distribution approaches the experimental values. Especially, the theoretical value with the third approximation shows a good agreement with the actual values for the whole probability distribution of noise annoyance.

\subsubsection{Comparison between multi-dimensional non- linear regression model and linear regression model}

The prediction results of the noise annoyance score by using the multi-dimension nonlinear regression model proposed in Section 2.1.2 were compared with the results by the standard linear regression model (i.e., autoregression (AR) model) ${ }^{8}$ The AR model considers only the liner correlation among instantaneous values of the noise annoyance and the sound pressure level. However, there are several linear and nonlinear correlations among the instantaneous values. Furthermore, since 


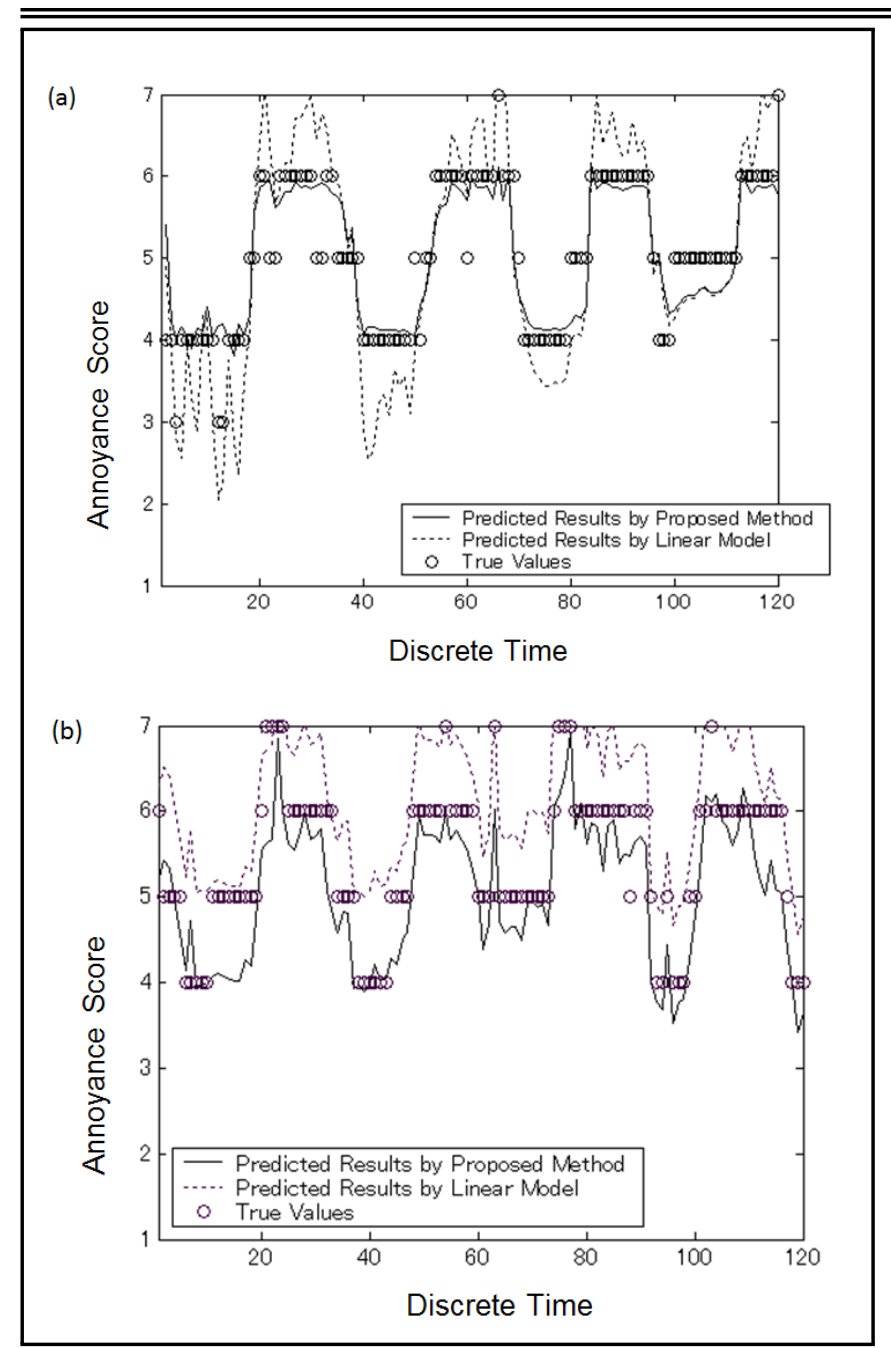

Figure 3. A comparison between the predicted results for noise annoyance by the proposed nonlinear regression model and the linear regression model. (a) Prediction result for data 1 ; (b) prediction result for data 2.

the relationship between the noise annoyance and the sound pressure level is nonlinear, liner regression model is not adequate for expressing the relationship. Therefore, a multidimensional nonlinear regression model was introduced. The prediction equations in Eq. (14) with $p=1$ and $p=2$ are expressed as follows:

$$
\begin{aligned}
& \hat{y}=\left\langle y \mid x_{1}\right\rangle=\sum y P\left(y \mid x_{1}\right)= \\
& =\frac{\sum_{m=0}^{1} \sum_{n=0}^{i} A_{m n} d_{1 m} \phi_{n}\left(x_{1}\right)}{\sum_{n=0}^{i} A_{0 n} \phi_{n}\left(x_{1}\right)}, \quad(p=1) \\
& \hat{y}=\left\langle y \mid x_{1}, x_{2}\right\rangle=\sum y P\left(y \mid x_{1}, x_{2}\right)= \\
& =\frac{\sum_{m=0}^{1} \sum_{n_{1}=0}^{i} \sum_{n_{2}=0}^{i} A_{m n_{1} n_{2}} d_{1 m} \phi_{n_{1}}\left(x_{1}\right) \phi_{n_{2}}\left(x_{2}\right)}{\sum_{n_{1}=0}^{i} \sum_{n_{2}=0}^{i} A_{0 n_{1} n_{2}} \phi_{n_{1}}\left(x_{1}\right) \phi_{n_{2}}\left(x_{2}\right)} \cdot \quad(p=2)
\end{aligned}
$$

The expansion coefficients were considered until the order $i(i=1,2, \cdots, 6)$ in Eq. (20) in the same manner as Section 3.1.1. After calculating expansion coefficients $A_{m n}$ and
Table 2. Prediction errors of the multi-dimensional nonlinear regression model and linear regression model. The cells with lowest prediction error are highlighted.

\begin{tabular}{|c|c|c|}
\hline $\begin{array}{c}\text { Order } \\
(i)\end{array}$ & \multicolumn{2}{|c|}{$\begin{array}{c}\text { Multi-dimensional } \\
\text { Nonlinear Regression } \\
\text { Model }\end{array}$} \\
\cline { 2 - 3 } & $p=1$ & $p=2$ \\
\hline 1 & 0.424 & 0.453 \\
\hline 2 & 0.423 & 0.401 \\
\hline 3 & 0.460 & 0.478 \\
\hline 4 & 0.459 & 0.469 \\
\hline 5 & 0.465 & 0.518 \\
\hline 6 & 0.464 & 0.518 \\
\hline \multicolumn{2}{|c|}{ Linear Regression Model } \\
\hline- & $p=1$ & $p=2$ \\
\hline- & 0.424 & 0.543 \\
\hline
\end{tabular}

(a) Prediction errors for data 1

\begin{tabular}{|c|c|c||}
\hline $\begin{array}{c}\text { Order } \\
(i)\end{array}$ & \multicolumn{2}{|c|}{$\begin{array}{c}\text { Multi-dimensional } \\
\text { Nonlinear Regression } \\
\text { Model }\end{array}$} \\
\cline { 2 - 3 } & $p=1$ & $p=2$ \\
\hline 1 & 0.551 & 0.602 \\
\hline 2 & 0.547 & 0.557 \\
\hline 3 & 0.622 & 15.7 \\
\hline 4 & 0.617 & 21.2 \\
\hline 5 & 0.625 & 10.3 \\
\hline 6 & 0.625 & 7.84 \\
\hline Linear Regression Model \\
\hline- & $p=1$ & $p=2$ \\
\hline- & 0.825 & 1.91 \\
\hline
\end{tabular}

(b) Prediction errors for data 2

$A_{m n_{1} n_{2}}$ in Eq. (20) by use of the learning data (i.e., data 1), the noise annoyance score was predicted based on the ob-servation of sound pressure level of data 1 and data 2 . The prediction results are shown in Table 2 (a) and Table 2 (b), respectively. The prediction errors are shown in this table. The case of taking into consideration of the expansion coefficients until $i=2$ shows the most accurate prediction among all cases, and the better prediction result is obtained in this case than the result by the standard linear regression model. Moreover, the proposed method of expansion series type sometimes shows worse prediction for accuracy in the case of considering some higher order expansion coefficients. The Akaikes information criterion (AIC) is well known to determine an optimal order of regression models. ${ }^{9}$ When calculating the expansion coefficients using finite number of the learning data, it is one of the future problems to find an optimal number of expansion terms by extending the AIC. The comparison between the prediction result of the proposed method with $p=1, i=2$ and the linear regression model is shown in Fig. 3. Data 1 and data 2 are sound pressure levels measured in two typical different situations of traffic flow. Data 1 was measured in a situation of light traffic flow (sound level range 55-90 dB), and data 2 was measured in a situation of heavy traffic flow (sound level range 70-100 dB). The two data sets appear to have quite similar varying patterns of the sound pressure level, since the traffic flow was affected roughly by the time period of a traffic signal existing near the 
Table 3. Prediction errors of multi-dimensional nonlinear regression model and linear regression model. The cells with lowest prediction error are highlighted.

\begin{tabular}{|c|c|c|}
\hline $\begin{array}{c}\text { Order } \\
(i)\end{array}$ & \multicolumn{2}{|c|}{$\begin{array}{c}\text { Multi-dimensional } \\
\text { Nonlinear Regression } \\
\text { Model }\end{array}$} \\
\cline { 2 - 3 } & $p=1$ & $p=2$ \\
\hline 1 & 4.00 & 3.63 \\
\hline 2 & 3.88 & 3.50 \\
\hline 3 & 3.35 & 3.44 \\
\hline 4 & 3.70 & 3.39 \\
\hline 5 & 3.36 & 3.10 \\
\hline 6 & 3.24 & 3.01 \\
\hline \multicolumn{3}{|c|}{ Linear Regression Model } \\
\hline- & $p=1$ & $p=2$ \\
\hline- & 3.27 & 4.01 \\
\hline
\end{tabular}

(a) Prediction errors for data 1 in $[\mathrm{dB}]$

\begin{tabular}{|c|c|c|}
\hline $\begin{array}{c}\text { Order } \\
(i)\end{array}$ & \multicolumn{2}{|c|}{$\begin{array}{c}\text { Multi-dimensional } \\
\text { Nonlinear Regression } \\
\text { Model }\end{array}$} \\
\cline { 2 - 3 } & $p=1$ & $p=2$ \\
\hline 1 & 9.06 & 8.88 \\
\hline 2 & 8.56 & 9.18 \\
\hline 3 & 7.83 & 8.49 \\
\hline 4 & 8.05 & 8.54 \\
\hline 5 & 7.79 & 8.05 \\
\hline 6 & 7.69 & 12.73 \\
\hline Linear Regression Model \\
\hline- & $p=1$ & $p=2$ \\
\hline- & 7.78 & 8.91 \\
\hline
\end{tabular}

(b) Prediction errors for data 2 in $[\mathrm{dB}]$

observation point. The proposed method can predict the noise annoyance more accurately than the standard linear regression model by choosing the expansion terms appropriately.

\subsection{Experimental Consideration for Prediction of Sound Pressure Level by Observing Noise Annoyance}

The comparison between the proposed multi-dimension nonlinear regression model and the linear regression model for the prediction of sound pressure level $x$ is considered in the same manner as Section 3.1.2. The multi-dimension nonlinear regression models with $p=1$ and $p=2$ are expressed as follows.

$$
\begin{aligned}
& \hat{x}=\frac{\sum_{m=0}^{1} \sum_{n=0}^{i} B_{m n} c_{1 m} \varphi_{n}(y)}{\sum_{n=0}^{i} B_{0 n} \varphi_{n}(y)}, \quad(p=1) \\
& \hat{x}=\frac{\sum_{m=0}^{1} \sum_{n_{1}=0}^{i} \sum_{n_{2}=0}^{i} B_{m n_{1} n_{2}} c_{1 m} \varphi_{n_{1}}\left(y_{1}\right) \varphi_{n_{2}}\left(y_{2}\right)}{\sum_{n_{1}=0}^{i} \sum_{n_{2}=0}^{i} B_{0 n_{1} n_{2}} \varphi_{n_{1}}\left(y_{1}\right) \varphi_{n_{2}}\left(y_{2}\right)} \cdot \quad(p=2)
\end{aligned}
$$

The comparison between the prediction results by using the proposed method and the linear regression model is shown in Table 3. Moreover, the prediction results of the proposed
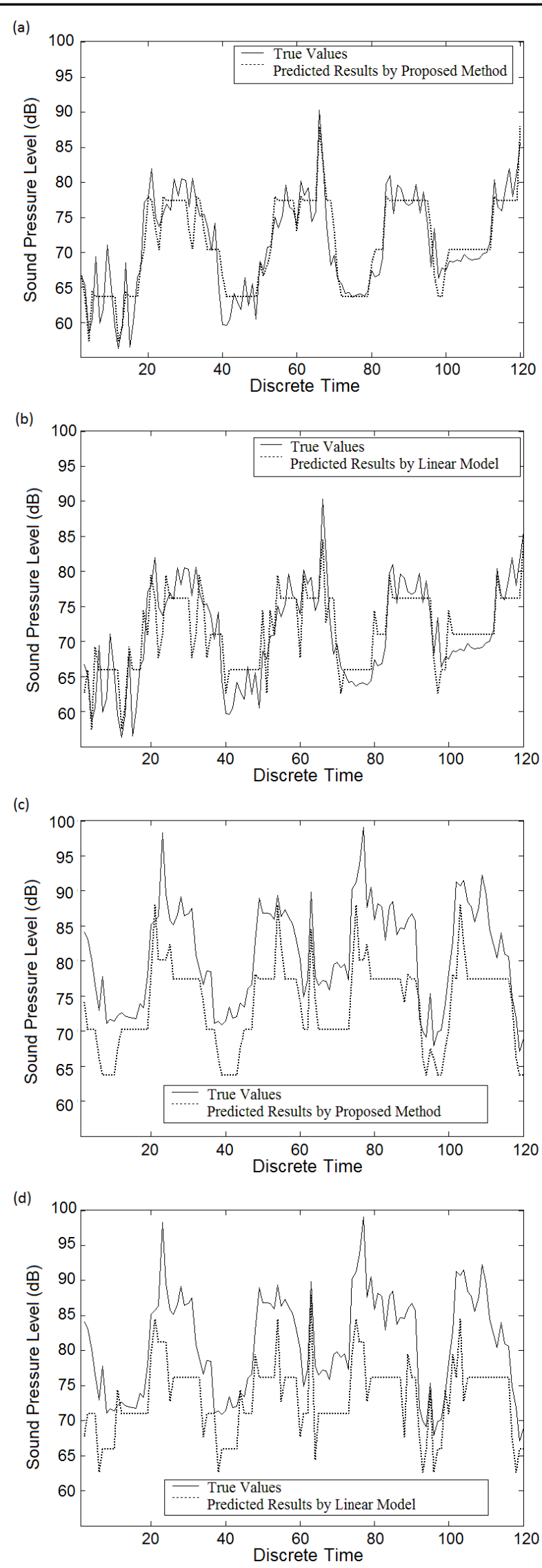

Figure 4. A comparison between the predicted results for sound pressure level by the proposed nonlinear regression model and the linear regression model. (a) Prediction result by proposed method for data 1; (b) Prediction result by linear model for data 1; (c) Prediction result by proposed method for data 2; (d) Prediction result by linear model for data 2 . 
method with $p=2, i=6$ and $p=2, i=5$ are shown in Fig. 4 (a) and Fig. 4 (b), respectively. By choosing the number of expansion terms appropriately, the proposed method shows more accurate prediction than the results of liner regression model for the sound pressure level.

\section{CONCLUSION}

In this paper, statistical methods for evaluating and predicting the relationship between sound pressure level and noise annoyance were considered. More specifically, a simple evaluation method for the relationship between the sound pressure level (objective physical quantity) and the noise annoyance (subjective amount of psychology) was derived based on the correlation infor-mation latent in both variables from the practical viewpoints. The theory was realized by introducing expansion series expression of probability distribution considering not only the lower order linear correlation but also the higher order nonlinear correlation information related to human sensitivity.

The methods for predicting the time course of fluctuation and the probability distributions of noise annoyance score from the observation data of sound pressure level were proposed by introducing the conditional probability distribution and the nonlinear regression model. Furthermore, the prediction methods for the sound pressure level based on the observation of noise annoyance were considered. Finally, the effectiveness of the proposed methods was investigated experimentally by applying it to the actual data of road traffic noise. The proposed approach is quite different from standard methods based on linear regression model, and it is still in the early stage of development of prediction method based on the correlation information of the sound pressure level and noise annoyance. Therefore, many practical problems are left to be considered in the future. For example, (i) the proposed method should be applied to the other actual data of sound environment, and its practical usefulness should be verified in each actual situation; (ii) the optimal order selection method for the statistical regression models based on the higher order correlation information should be investigated by considering the complexity of a phenomenon and the number of data which can be used; and (iii) the proposed theory should be extended to the actual situation under existence of the external noise.

\section{REFERENCES}

1 Yamaguchi, S., Kato, T., Oimatsu, K. and Saeki, T. A psychological evaluation method for fluctuating random noise based on fuzzy set theory, Applied Acoustics, 45, 139-154, (1995).

2 Yamaguchi, S. and Saeki, T. A practical prediction method of psychological response to arbi-trary non-white random noise based on simplified patterns of membership functions, Applied Acoustics, 48, 155-174, (1996).

3 Ikuta, A., Ohta, M. and Siddique, M. N. H. Prediction of probability distribution for the psy-chological evaluation of noise in the environment based on fuzzy theory, International J. of Acoust. and Vib., 10, 107-114, (2005).

4 Ikuta, A., Masuike, H. and Ohta, M. State estimation for sound environment system with un-known structure by introducing fuzzy theory, IEEJ Trans. EIS, 127, 770-777, (2007) (in Japanese).

5 Ohta, M. and Ikuta, A. A basic theory of statistical generalization and its experiment on the multi-variate state for environmental noiseA unification on the variate of probability func-tion characteristics and digital or analogue type level observation, J. Acoust. Soc. Japan, 39, 592-603, (1983) (in Japanese).

6 Kuwano, S. and Namba, S. Continuous judgment of levelfluctuating sounds and the relationship between overall noise annoyance and instantaneous noise annoyance, Psychological Research, 47, 27-37, (1985).

7 Ohta, M. and Koizumi, T. General statistical treatment of the response of nonlinear rectifying device to a stationary random input, IEEE Trans. On Information Theory, 14, 595-598, (1968). http://dx.doi.org/10.1109/tit.1968.1054178

8 Franses, P. H. Time series models for business and economic forecasting, Cambridge Univer-sity Press, (1998)

9 Akaike, H. Information theory and an extension of the maximum likelihood principle, 2nd In-ter. Symp. On Information Theory, Akademiai Kiado, Budapest, 267-281, (1973). http://dx.doi.org/10.1007/978-1-4612-1694-0_15 